\title{
ESTUDO ARQUEOBOTÂNICO DO CASTRO DE CIDADELHE (MESÃO FRIO): RESULTADOS PRELIMINARES
}

\author{
FILIPE COSTA VAZ ${ }^{(1)}$, JOÃO PEDRO TERESO ${ }^{(1)}$, PAULO PINHO LEMOS $^{(2)} \&$ PAULA BARREIRA ABRANCHES $^{(2)}$
}

Resumo:

Abstract:

From October to December 2013, several soil samples were recovered during the archaeological excavations of the settlement of Cidadelhe (Mesão Frio, Northern Portugal) in order to undergo archaeobotanical analysis. These samples are originated from two separate groups of contexts: one prior to the construction of the settlement's wall (Phase $1-8^{\text {th }}$ to the $9^{\text {th }}$ century BC) and the remaining in the reinforced buttress of the wall, built during the Roman period (Phase II).

Despite the reduced excavation area, this archaeobotanical study allowed the identification of two distinct ecological moments, both with strong anthropic influences and closely related to the archaeological phases recorded on site. The first and older phase, prior to the construction of the wall, refers to an environment with the presence of herbaceous ruderal species, ferns, deciduous and evergreen oaks. The second phase heavily contrasts with the latter, with the presence of a much wider range of species such as cereals (emmer and broomcorn millet) and a set of species (chestnut, walnut, fig tree and vine tree) which strongly infer their active cultivation.

Keywords: Iberian NW, Iron Age, Archaeobotany, Charcoal analysis, Carpology

\section{INTRODUÇÃO}

Localizado na margem direita do rio Douro, o Castro de Cidadelhe (Fig. 1 - 41 ${ }^{\circ} 10^{\prime} 42,85^{\prime}$ ' N; $07^{\circ} 50^{\prime} 39,50^{\prime}$ 'O) é parte integrante da freguesia de Cidadelhe, concelho de Mesão Frio, distrito de Vila Real, encontrando-se classificado como Imóvel de Interesse Público, estando afeto à Direção Regional de Cultura do Norte (DRCN), para efeitos de gestão.

$\mathrm{O}$ projeto de Conservação e Restauro da Muralha do Castro de Cidadelhe, desenvolvido pela DRCN, foi executado no último trimestre de 2013, incluiu a realização de sondagens arqueológicas de avaliação (num total de $161 \mathrm{~m} 2$ ), com o intuito de confirmar a natureza, tipo e extensão das fundações do troço de muralha exterior, avaliando simultaneamente o seu estado de conservação.
Subsequentemente, e ao abrigo da colaboração estabelecida entre a Archeo'Estudos Lda. e o Centro de Investigação em Biodiversidade e Recursos Genéticos - Universidade do Porto (CIBIO-UP), através do grupo Environmental Archaeology (EnvArch), as amostras sedimentares recolhidas ao longo da sequência estratigráfica identificada na Sondagem 1 (Fig. 2) foram estudadas nas suas componentes antracológica e carpológica com o objetivo de ajudar à interpretação do contexto construtivo desta estrutura e de caracterizar o ambiente paleopaisagístico envolvente. O presente artigo versará sobre os resultados arqueobotânicos cujos dados obtidos mereceram algumas considerações no quadro do norte português.

\footnotetext{
(1) EnvArch - Environmental Archaeology Research Group, CIBIO - Research Center in Biodiversity and Genetic Resources, Universidade do Porto, Campus Agrário de Vairão, Rua Padre Armando Quintas, nº 7, 4485-661 Vairão, Portugal. E-mail: filipe.mcvaz@gmail.com, jptereso@gmail.com

(2) Archeo'Estudos, Investigação Arqueológica, Lda. Rua da Igreja, 226, 4415-937 Seixezelo, Vila Nova de Gaia, Portugal. E-mail: paplemos@gmail.com, paula@archeoestudos.com.

${ }^{1}$ Projeto desenvolvido no âmbito de uma candidatura do QREN, ON2, Programa Turismo Douro Infraestrutural, intitulada "De Aliobriga a Cidadelhe", tendo como promotor a Direção Regional de Cultura do Norte. Execução - Archeo'Estudos, Investigação Arqueológica Lda; Topografia e Levantamentos Fotogramétricos - Hugo Pires; Empreitada - Assinatura de Modernidade.
} 


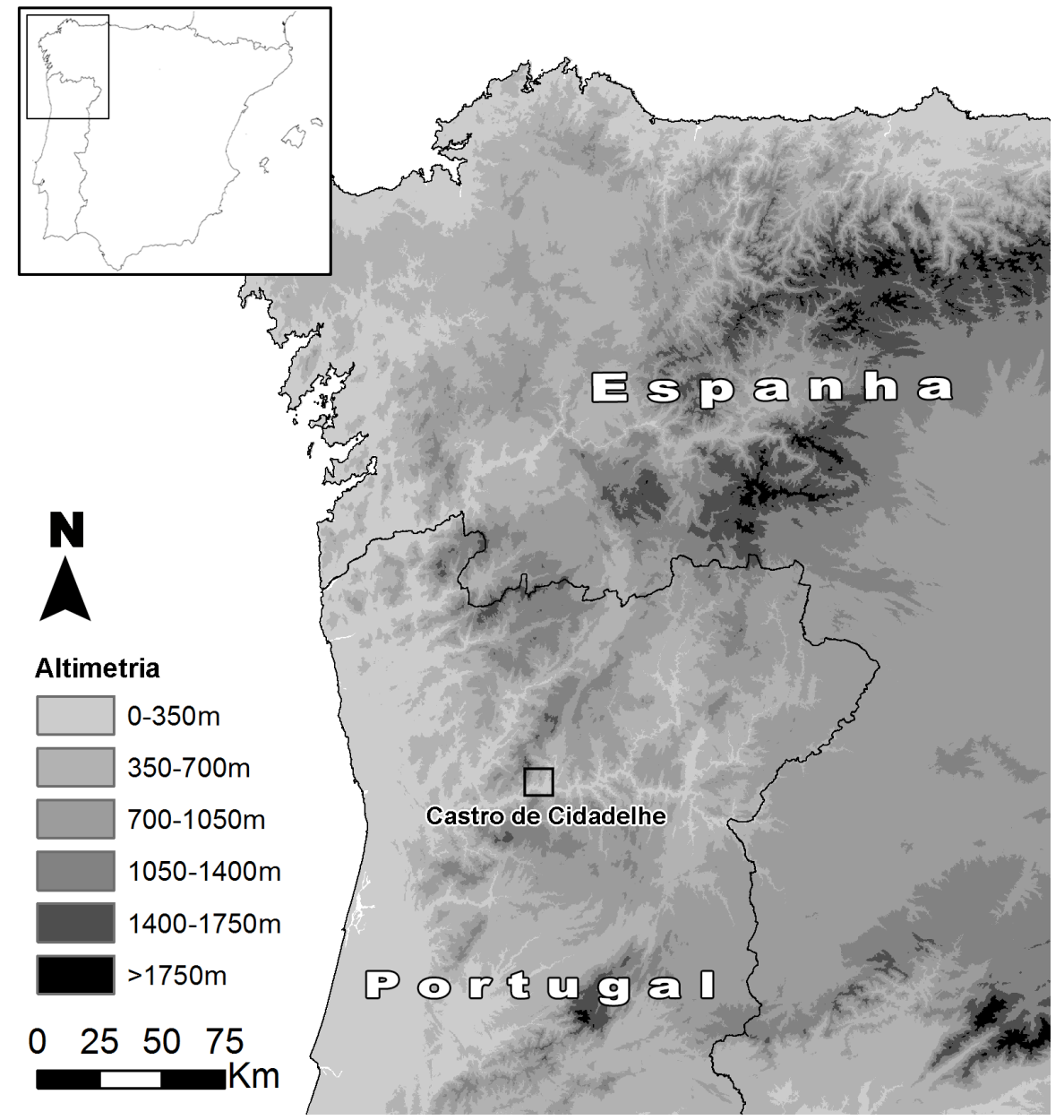

Fig. 1. Localização do Castro de Cidadelhe no norte de Portugal Fig. 1. Location of Castro de Cidadelhe in the north of Portugal.

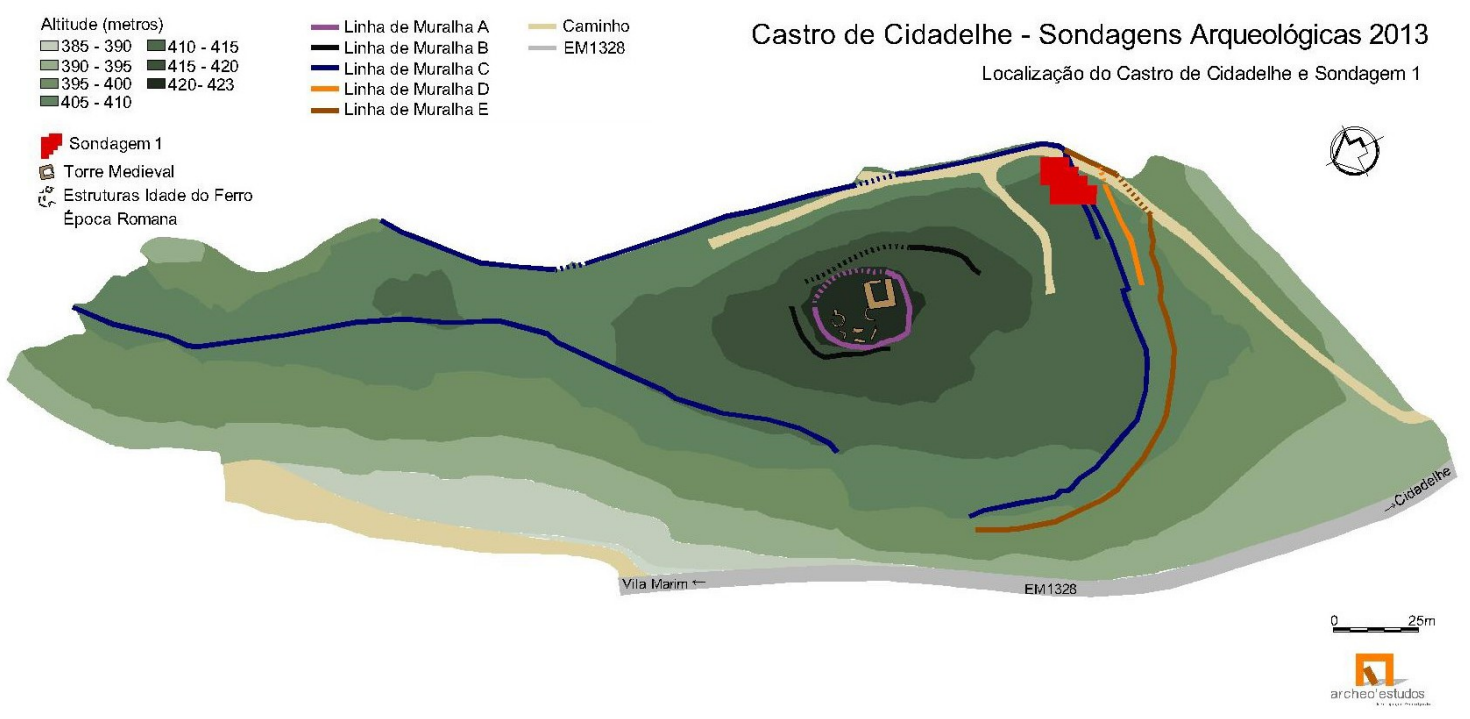

Fig. 2. Posicionamento da sondagem 1 e principais estruturas na planta do povoado.

Fig. 2. Location of test pit 1 in the analytical blueprint of the Castro. 


\section{O SÍTIO E A ESCAVAÇÃO}

O Castro de Cidadelhe é um dos sítios arqueológicos que, da Idade do Ferro à Alta Idade Média, atesta a remota ocupação do vale do Douro, onde o relevo desempenhou um papel preponderante na sua organização espacial. $O$ assentamento deste sitio foi fortemente condicionado por critérios defensivos, pelas condições orográficas e pela necessidade que as populações sentiram em colmatar as fragilidades do terreno natural.

Os primeiros trabalhos científicos levados a cabo no Castro de Cidadelhe foram conduzidos por Armando Coelho Ferreira da Silva, entre 1983 e 1985. Esta intervenção, centrada no interior da acrópole, revelou uma sequência de níveis castrejos e romanos, sob uma estrutura quadrangular que corresponderá a uma torre medieval (SILVA 1986). Esta torre estará relacionada com a fortificação da civitate de Aliovirio, instituída pelo monarca asturiano Afonso III, nos primórdios do movimento de Presúrias (LiMA 2010-2011).

$\mathrm{Na}$ sequência da prospeção arqueológica sistemática realizada em 2013, foram identificadas cinco possíveis linhas de muralha neste povoado fortificado (Fig. 2 e 3). As linhas de muralha A e B localizam-se na acrópole, tendo sido edificadas entre 138 a.C. e a segunda metade século I d.C. (SILVA 1986). A linha de muralha C (Fig. 2) corresponde à muralha exterior do povoado, visível numa extensão de $575 \mathrm{~m}$, cuja primeira etapa de construção terá ocorrido entre 500 e 200 a.C., enquanto a segunda se situará entre 138 a.C. e segunda metade do século I d.C. (SILVA 1986). As linhas de muralha D e E encontram-se localizadas a Sul da Linha de Muralha C, paralelamente à mesma, não havendo ainda dados suficientes para o seu enquadramento cronológico seguro.

As sondagens arqueológicas, executadas também em 2013, distribuíram-se unicamente ao longo do troço de muralha $\mathrm{C}$, que foi alvo de ações de estabilização e consolidação. Na sondagem 1 (Fig. 2 e 4) foram identificados três momentos construtivos, cujo intuito era reforçar a linha de muralha funcionando, no seu conjunto, como um contraforte, com uma espessura superior a $1.30 \mathrm{~m}$, composta por três troços sobrepostos, com orientações diferentes, assentando diretamente numa camada argilosa. Estes troços eram formados por diversas unidades compostas exclusivamente de pedras, alternando com níveis constituídos unicamente por terra. As restantes três sondagens confirmaram a complexidade desta estrutura defensiva, revelando de igual forma várias fases e soluções construtivas.

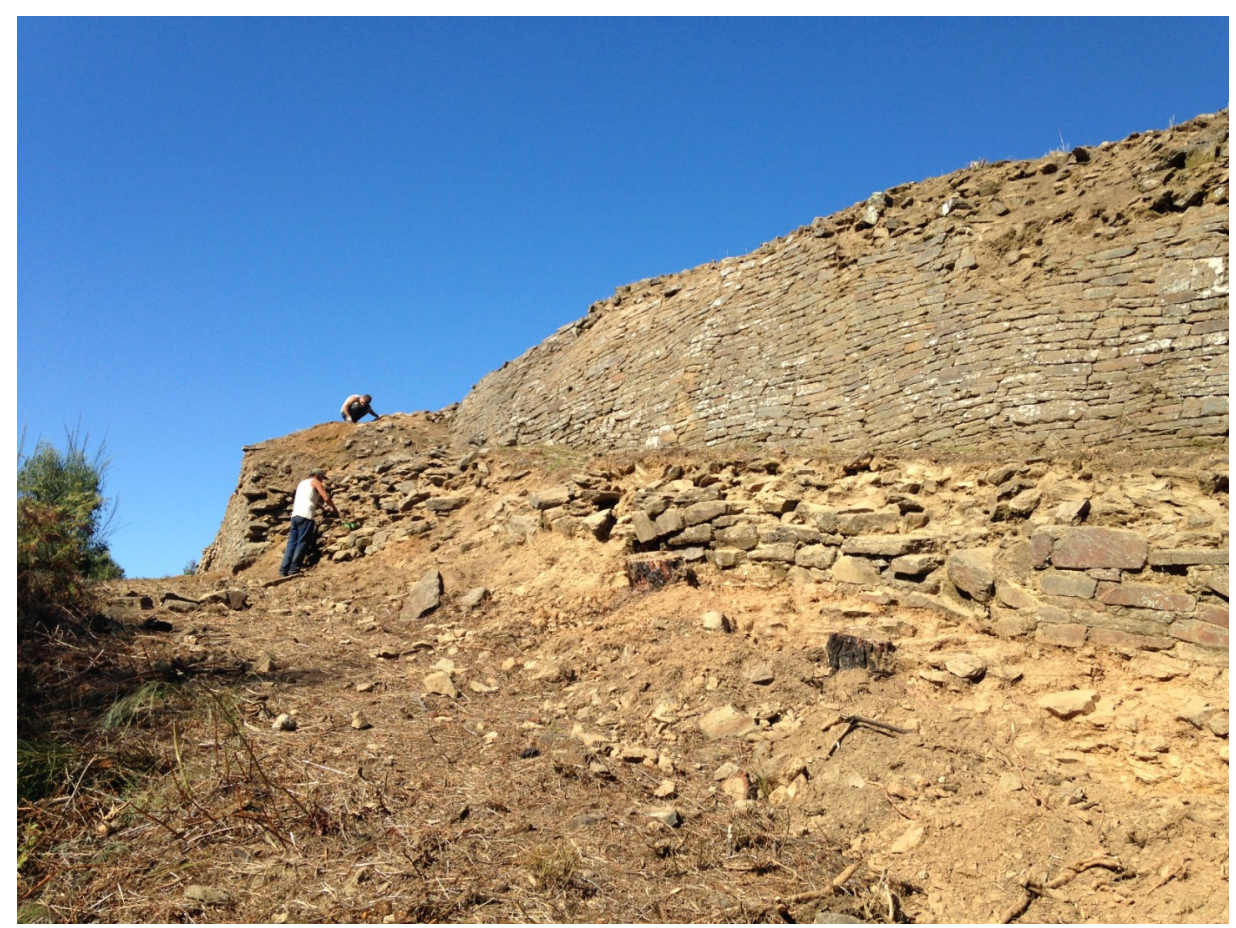

Fig. 3. Troço da Muralha $\mathrm{C}$ em trabalhos de restauro.

Fig. 3. Wall C during restoration procedures. 


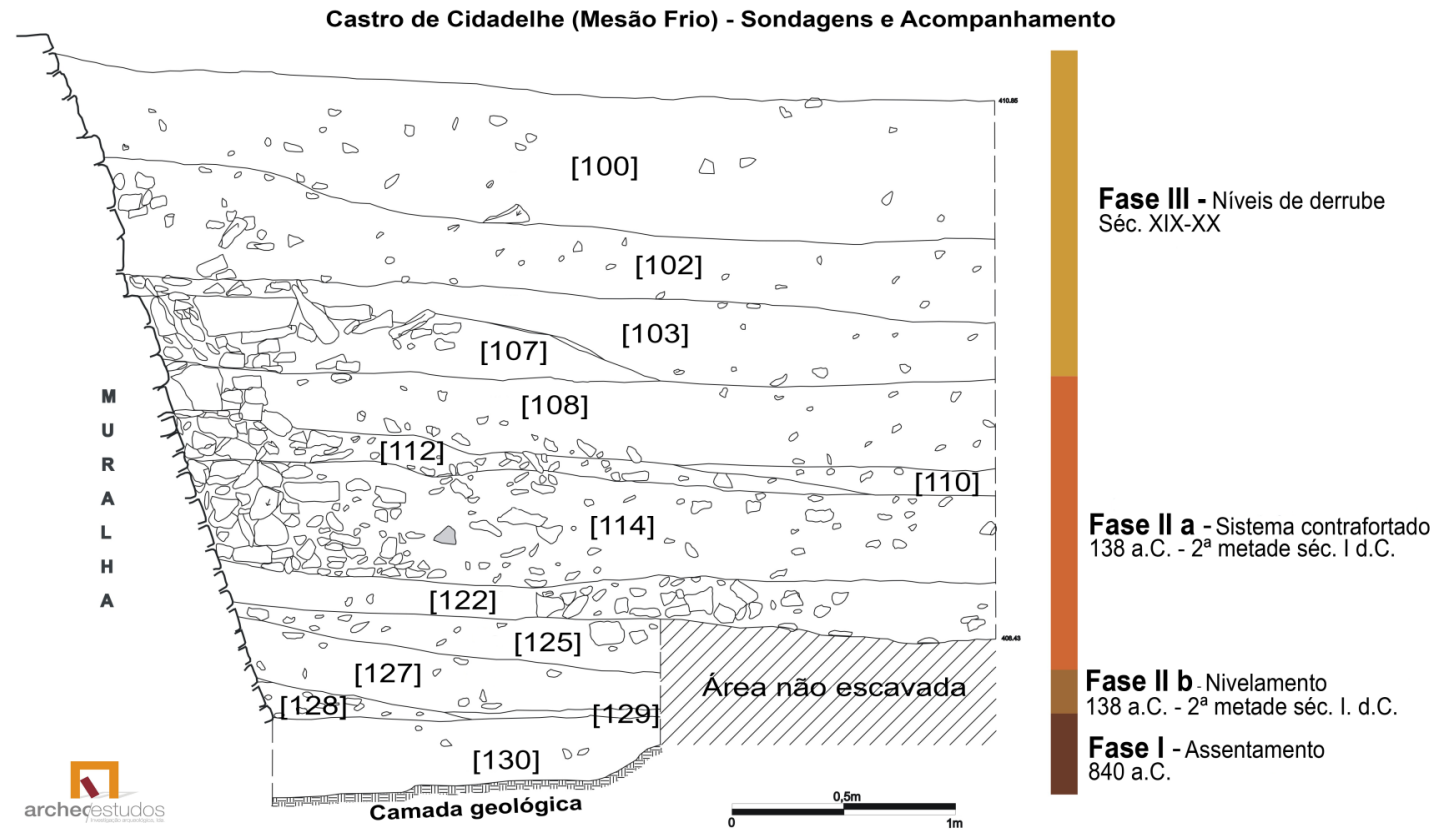

Fig. 4. Corte Oeste da Sondagem 1.

Fig.4. West section of Trench 1.

\section{MATERIAIS E MÉTODOS}

\subsection{Os contextos}

No decorrer dos trabalhos arqueológicos na sondagem 1 - aberta numa área contígua ao troço de muralha $\mathrm{C}$ na zona sul do castro de Cidadelhe (Fig.2) - foram efetuadas recolhas de sedimentos de forma pontual, assim como recolhas manuais. Com um volume total de 122 litros, estes sedimentos foram recolhidos em 11 unidades estratigráficas [u.e.] identificadas ao longo de uma sequência vertical de 3.26 metros de profundidade na qual foram identificadas duas fases de ocupação de Cidadelhe (Fig. 4).

Adscrita à Fase I, foram recolhidas 4 amostras na u.e. [130] (Tab. 1), correspondendo a um nivelamento do substrato geológico com o propósito de preparar a construção da muralha. As 4 amostras recolhidas respeitam a uma recolha sedimentar $(11 \mathrm{~A})$ realizada no topo da unidade e a 3 recolhas manuais em concentrações de carvões (12A, 13A, 14A) mas a uma cota inferior.

Foi realizada uma datação $\mathrm{C}^{14}$ num carvão recolhido na amostra $12 \mathrm{~A}$, que balizou cronologicamente esta unidade entre o 975 e 830 a. $C^{2}$. No entanto, o carvão que foi alvo de datação não foi sujeito a uma identificação prévia. Posteriormente verificouse que a totalidade dos carvões estudados nesta amostra provieram da carbonização de um fragmento de madeira de Quercus caducifólio (vide Resultados e Discussão) - táxon que abarca espécies de grande longevidade. Para mais, estes carvões ostentavam anéis de crescimento com curvaturas pouco pronun- ciadas, potencialmente indicadoras de uma árvore de grande porte. Estes indícios aumentam a possibilidade de existência de um efeito de madeira-antiga (LÓPEZ-DÓRIGA 2014) que dificulta a interpretação da análise radiométrica. De qualquer forma, considerando a integração estratigráfica desta unidade anterior à construção da muralha - a indicação cronológica da datação enquadra o conjunto arqueobotânico nela presente e não a edificação da referida estrutura. Ou seja, constitui-se efetivamente como uma indicação ante quem da ocupação plena do local.

Nas unidades estratigráficas relativas à Fase II foram recolhidas 10 amostras num total de 99 litros de sedimento procedente de 10 u.e. (Tab. 1). Associadas a uma sequência de elementos construtivos, estes contextos organizam-se em três tipos:

- u.e. [127], [128] e [129] - conjunto estratigráfico interpretado como servindo de nivelamento e base para o assentamento do sistema contrafortado;

- u.e. [108] a [111], [114] e [125] - pacote sedimentar com depósitos muito semelhantes entre si, depositados intencionalmente como reforço do sistema contrafortado;

- u.e. [121] - corresponde a um nível de sedimento nos interstícios do miolo da muralha.

Não foram realizadas datações nestas unidades e o espólio cerâmico recolhido foi escasso e inconclusivo. Como tal, não foi possível definir uma cronologia circunscrita associada a estes contextos, devendo-se integrar-se na fase de ocupação romana deste castro.

${ }^{2}$ Beta Analytic Miami, Beta-372845: 2760+/30 BP. Resultados calibrados 2 Sigma com 95\% de probabilidade: Cal BC 975 a 830 (Cal BP 2925 a 2780 ). 
Tab. 1. Amostras analisadas e respetiva informação contextual e de recolha.

Tab. 1. Samples recovered and their respective methodological background.

\begin{tabular}{|c|c|c|c|c|c|}
\hline Amostra & Volume (I) & UE & Tipo de recolha & Tipo de Contexto & Fase \\
\hline $1 \mathrm{~A}$ & 10 & $108 / 109$ & \multirow{11}{*}{$\begin{array}{l}\text { Amostra } \\
\text { sedimentar }\end{array}$} & \multirow{6}{*}{$\begin{array}{l}\text { Sedimentos de reforço do sistema } \\
\text { de contrafortagem }\end{array}$} & \multirow{10}{*}{ Fase I } \\
\hline $2 A$ & 10 & $108 / 109$ & & & \\
\hline $3 A$ & 10 & $110 / 111$ & & & \\
\hline $4 \mathrm{~A}$ & 10 & $110 / 111$ & & & \\
\hline $5 A$ & 10 & 114 & & & \\
\hline $7 \mathrm{~A}$ & 10 & 125 & & & \\
\hline $6 \mathrm{~A}$ & 10 & 121 & & Intersticios da muralha & \\
\hline $8 \mathrm{~A}$ & 10 & 127 & & \multirow{3}{*}{$\begin{array}{l}\text { Sedimentos de assentamento do } \\
\text { sistema de contrafortagem }\end{array}$} & \\
\hline $9 A$ & 9 & 128 & & & \\
\hline $10 \mathrm{~A}$ & 10 & 129 & & & \\
\hline $11 \mathrm{~A}$ & 17 & \multirow{4}{*}{130} & & \multirow{4}{*}{$\begin{array}{l}\text { Depósitos de nivelamento do } \\
\text { geológico }\end{array}$} & \multirow{4}{*}{ Fase I } \\
\hline $12 \mathrm{~A}$ & 1 & & \multirow{3}{*}{ Manual } & & \\
\hline $13 \mathrm{~A}$ & 4 & & & & \\
\hline $14 \mathrm{~A}$ & 1 & & & & \\
\hline
\end{tabular}

\subsection{Metodologia Laboratorial}

As 14 amostras foram processadas pela equipa de arqueólogos através do método de flutuação manual simples, com recurso a uma malha de $0,25 \mathrm{~mm}$. A fração leve foi triada com recurso a uma lupa binocular, tendo-se procedido à separação da componente carpológica. Os frutos e sementes foram observados à lupa binocular e o diagnóstico efetuou-se por comparação morfológica com elementos atuais, com recurso às coleções de referência do herbário da Universidade do Porto e do CIBIO e com atlas anatómicos (e.g. BERGGREN 1981; ANDERBERG 1994; JACOMET 2006) e outras obras da especialidade (HILLMAN et al. 1996; BuXó 1997a). No caso das cariopses, foram consideradas unidades todas aquelas, inteiras ou fragmentadas, que apresentavam o escutelo.

No que respeita ao estudo antracológico, os fragmentos de carvão de dimensões superiores a $2 \mathrm{~mm}$ foram fracturados manualmente segundo as três secções de diagnóstico: transversal, radial e tangencial. A observação foi realizada com recurso a uma lupa binocular e a um microscópio ótico de luz refletida. O diagnóstico foi efetuado com recurso a atlas anatómicos e morfológicos (e.g. SCHWEINGRUBER 1990a, 1990b; VERNET et al. 2001) e a ferramentas interativas de apoio à identificação das espécies (HEISS 2002). Para a identificação de fragmentos de Ericales recorreu-se ao estudo específico de P. Queiroz e C. Van der Burgh (1989), sendo que, no caso do género Erica optou-se pela utilização dos tipos morfológicos definidos por J. Tereso (2007).

Para além da identificação, registaram-se também um conjunto de atributos dendrológicos capazes de fornecer importantes conclusões relativamente à gestão e uso destas madeiras, tais como curvatura dos anéis, existência de vitrificação e fissuras radiais, entre outras.

A curvatura dos anéis é medida de acordo em três níveis (acentuada, moderada e fraca) e permite perceber qual o porte mínimo do ramo ou tronco de onde proveio o carvão (CARRIÓN 2003, 2007; MARGUERIE \& HUNOT 2007), dando informações quanto a possíveis tendências na recole- ção destes recursos lenhosos.

Comumente designada por vitrificação, a fusão dos tecidos lenhosos é uma alteração anatómica cujas causas ainda não estão completamente definidas. No entanto, aponta-se como possível causa uma conjuntura de fatores relativos à biologia das espécies (índices de humidade e quantidade de seiva, etc.) e à verificação de elevadas temperaturas durante a combustão (CARRIÓN 2003; 2007; MARGUERIE \& HUNOT 2007; MACPARLAND et al. 2010; HENRY 2011), ainda que não seja claro o que efetivamente causa este fenómeno.

Relativamente comuns em carvões, as causas para a ocorrência de fissuras radiais não está ainda completamente definida. Poderão ser o resultado de uma evaporação rápida durante o processo de combustão, fruto de um aquecimento brusco da humidade presente em lenhas frescas (CARRIÓN 2003, 2007; MARGUERIE \& HUNOT 2007; THÉRY-PARISOT et al. 2012).

Foi estabelecido previamente um mínimo de 100 fragmentos de carvões analisado por amostra. No entanto, se no intervalo entre os 50 e os 100 carvões surgisse um novo taxon ou morfotipo na amostra, seriam analisados mais 50, repetindo o procedimento sucessivamente. Na eventualidade das amostras não possuírem o número mínimo estabelecido, todos os carvões nela presentes seriam analisados. No entanto, apenas nos casos das amostras 1A, $6 \mathrm{~A}$ e $12 \mathrm{~A}$ - devido ao reduzido número de carvões existente - e no caso da amostra 9A - devido à pouca diversidade e estado de conservação dos carvões, foi identificado um número igual ou inferior ao mínimo definido metodologicamente.

\section{RESULTADOS}

\subsection{Carpologia}

Foram identificados 738 vestígios carpológicos (unidades e fragmentos) em 10 amostras recolhidas em Cidadelhe (Tab. 2), já que as restantes 4 amostras não forneceram qualquer vestígio carpológico. Contudo, c.88\% (647 macrorrestos) estava 
concentrado numa só amostra (11A). Com exceção do material recolhido nesta amostra, que se encontrava em bom estado de preservação, os frutos e sementes recolhidos nas restantes encontravam-se deteriorados, dificultando a sua identificação. Assim se justifica que algumas amostras (1A a 5A) sejam dominadas por fragmentos de taxonomia indeterminada.
Das 4 amostras sedimentares recolhidas na UE [130] (associada à Fase I), somente aquela recolhida na cota superior (11A) forneceu material carpológico em quantidade significativa. As amostras $12 \mathrm{~A}$ e $14 \mathrm{~A}, 24 \mathrm{~cm}$ e $38 \mathrm{~cm}$ abaixo da cota de recolha da amostra 11A, não forneceram vestígios carpológicos.

Tab. 2. Resultados do conjunto carpológico.

Tab. 2. Carpological results.

\begin{tabular}{|c|c|c|c|c|c|c|c|c|c|c|}
\hline Amostra & 1A & $2 \mathrm{~A}$ & $3 A$ & 4A & $5 A$ & 7A & 9A & $10 \mathrm{~A}$ & \multirow{3}{*}{\multicolumn{2}{|c|}{\begin{tabular}{c|}
$11 \mathrm{~A}$ \\
I $13 \mathrm{~A}$ \\
$\begin{array}{c}\text { Depósitos de nivel } \\
\text { do geológico }\end{array}$ \\
\end{tabular}}} \\
\hline Fase & \multicolumn{8}{|c|}{ II } & & \\
\hline Contexto & \multicolumn{6}{|c|}{ Reforço sistema de contrafortagem } & \multicolumn{2}{|c|}{$\begin{array}{l}\text { Assentamento do } \\
\text { sist. contrafortagem }\end{array}$} & & \\
\hline U.E. [ & 108 & 109 & 110 & 111 & 114 & 125 & 128 & 129 & & \\
\hline \multicolumn{11}{|c|}{ Cultivos } \\
\hline Panicum miliaceum & & & & & & 1 & & & & \\
\hline Triticum dicoccum (base de gluma) & & & 1 & & & 1 & & & & \\
\hline Triticum & & & & & & & & 1 & & \\
\hline Triticeae (frag.) & 1 & & & & & 3 & 2 & 2 & & \\
\hline \multicolumn{11}{|c|}{ Silvestres } \\
\hline cf. Asteraceae & & & & & & & & & 2 & \\
\hline Carex tipo muricata & & & & & & & & & 6 & \\
\hline Daphne gnidium & & & & & & & & & 1 & \\
\hline Galium aparine & & & & & & 1 & & & 11 & \\
\hline Galium aparine (frag.) & & & & & & & & & 14 & \\
\hline Gramineae & & & & & & & & & 3 & \\
\hline Hyoscyamus niger & & & & & & 2 & & & & \\
\hline Leguminosae - cf. Genisteae & & & 5 & & & & & & 5 & \\
\hline Leguminosae & & & 5 & 1 & & & 1 & & 12 & \\
\hline Leguminosae - Tipo Vicia sativa/angustifolia & & & & & & & & & 6 & 1 \\
\hline Leguminosae - Medicago & & & & & & & & & 19 & \\
\hline Polygonaceae - lenticular (frag.) & & & & & & & & & 37 & \\
\hline Polygonaceae - trigonal & & & & & & & & & & 1 \\
\hline Polygonum aviculare & & & & & & & & & 144 & \\
\hline Polygonum persicaria & & & & & & & & & 70 & \\
\hline Ranunculus & & & & & & & & & 9 & \\
\hline Rubus fruticosus - coll & & & 1 & & & 1 & & & 154 & \\
\hline Rubus fruticosus - coll (frag. c. 1/2) & & & & & & & & & 57 & \\
\hline Rubus (frag. Pequenos) & & & & & & & & & 58 & \\
\hline Solanum & & & & & & & & & 1 & \\
\hline Stellaria media & & & & & & & & & 9 & \\
\hline cf. Urtica & & & & & 1 & & & & & \\
\hline Verbena officinalis & & & & & & & & & 2 & \\
\hline Indeterminado (unidade) & & 3 & & & & & & & 13 & \\
\hline Indeterminado (frag.) & 5 & 9 & 27 & 1 & 12 & & & 1 & 9 & 1 \\
\hline Indeterminado - espinhos & & & & & & & & & 5 & \\
\hline \multicolumn{11}{|c|}{ Outros vestígios } \\
\hline Coprólitos & & & & & & & & & 12 & \\
\hline Aglomerados de coprólitos de térmitas & & & & & 6 & & & & & \\
\hline
\end{tabular}

Entre os vestígios carpológicos da amostra 11A (Tab. 2), salientam-se, pela sua abundância, os aquénios de Rubus fruticosus coll. (Fig. 5) (= tipo Rubus ulmifolius - tipo morfológico que inclui várias espécies de Rubus) e os aquénios de Polygonaceae (Polygonum aviculare, Polygonum persicaria e aquénios mal preservados identificados ao nível da família). Seguem-se, em abundância, os mericarpos de Galium aparine, as sementes de leguminosas silvestres, em especial Medicago, sementes de Stellaria media e aquénios de
Ranunculus. A interpretação de um conjunto tão grande de material carpológico só poderá ser feito por analogia com a ecologia atual dos taxa identificados. Esta tarefa é dificultada pelo facto de alguns diagnósticos taxonómicos se quedarem pelo nível género, tribo ou família, e também por diversos taxa apresentarem significativa amplitude ecológica.

A amostra 13A, recolhida $34 \mathrm{~cm}$ mais abaixo apresentava somente um fruto, uma semente e um fragmento indeterminado (Tab. 2). 


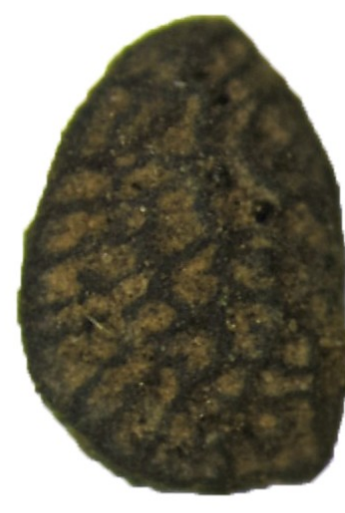

Fig. 5. Semente de Rubus fruticosus. Escala: $500 \mu \mathrm{m}$. Fig. 5. Seed of Rubus fruticosus. Scale: $500 \mu \mathrm{m}$.

Relativamente às amostras recolhidas em unidades cronologicamente enquadráveis na Fase II, das 2 são provenientes de depósitos associados a sedimentos do sistema de contrafortagem da muralha, destacando-se a presença de vestígios de cereais, ainda que parcos. Trata-se de um grão de trigo (Triticum sp.) e 4 pequenos fragmentos de cereais indeterminados.

Já nos sedimentos de reforço do sistema de contrafortagem foram identificados grãos de cereais, nomeadamente 1 grão de milho-miúdo (Panicum miliaceum) (Fig. 6) e 4 fragmentos de cereais indeterminados. Trata-se de fragmentos de grãos longos da tribo Triticeae, o que exclui as Panicoideae, as quais incluiem o milho-miúdo. Identificaram-se duas bases de gluma em bom estado de preservação que foi possível identificar como Triticum turgidum subsp. dicoccum. As bases de gluma são subprodutos do processamento do cereal, que podem incorporar os sedimentos arqueológicos em muitos tipos de contextos. Também nas amostras do sistema contrafortado surgiram vestígios carpológicos de plantas silvestres, ainda que em pouca quantidade. Salientam-se as sementes de leguminosas silvestres e de Hyoscyamus niger.

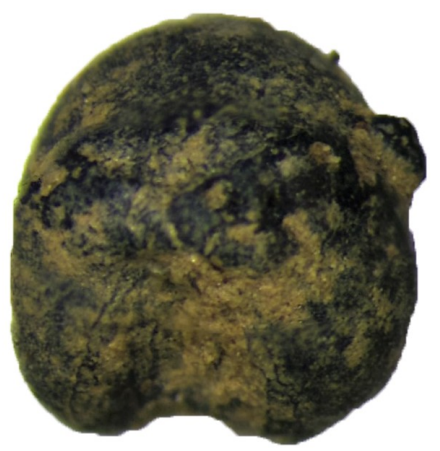

Fig. 6. Cariopse de Panicum miliaceum. Escala: $500 \mu \mathrm{m}$. Fig. 6. Grain of Panicum miliaceum. Scale: $500 \mu \mathrm{m}$.

\subsection{Antracologia}

Relativamente à Fase I, o leque de taxa e tipos anatómicos identificados no conjunto antracológico analisado foi relativamente reduzido (Tab. 3), tendo-se evidenciado um predomínio de Quercus de folha caduca (carvalho alvarinho ou cerquinho) e Quercus de folha perene (grupo no qual se incluem Quercus ilex (azinheira), Quercus suber (sobreiro) e o Quercus coccifera (carrasco). Com menor peso, surgiram Pteridium aquilinum (feto) (Fig. 7), Leguminosae (tojo, giestas, codeços, etc), Arbutus unedo (medronheiro) e uma ocorrência de Salix/Populus (salgueiro/choupo). É importante referir que Quercus de folha perene só é predominante numa das amostras recolhidas (13A) na qual é também o único tipo anatómico identificado.

Em oposição à fase anterior, as 10 amostras recolhidas em contextos da Fase II apresentam uma maior diversidade de taxa e tipos anatómicos (Tab. 3).

No conjunto de sedimentos de assentamento do sistema de contrafortagem registaram-se, por ordem de abundância, a presença de Quercus caducifólia e perenifólia, Leguminosae, Arbutus unedo, Quercus suber, Sambucus sp. (sabugueiro) e uma ocorrência de Cistus sp. (esteva) e de Taxus baccata (teixo).

As 6 amostras recolhidas no reforço do sistema de contrafortagem foram as que forneceram mais material antracológico passível de identificação (979 fragmentos). Registam-se novamente a presença de Quercus de folha caduca e perene mas em menor número do que o verificado noutros contextos, face ao total identificado. Seguem-selhe Leguminosae, Fraxinus sp. (freixo) e algumas ocorrências escassas de Rosaceae Maloidea [subfamília na qual se incluem Sorbus aucuparia (sorveira), Crataegus monogyna (pilriteiro) e Malus sylvestris (macieira) entre outras espécies silvestres e domésticas], Arbutus unedo, Cistus sp., Erica sp. (urzes), Ulmus sp. (ulmeiro) e Laurus sp. (loureiro). Em destaque pela sua raridade em contextos romanos no $\mathrm{NO}$ peninsular (vide infra), regista-se uma assinalável presença de Castanea sativa (castanheiro), Ficus carica (figueira) e Juglans regia (nogueira) e em menor número, Vitis vinifera (videira) (Fig. 8).

O último contexto amostrado (UE [121]) referente ao troço de muralha, e como seria expectável, forneceu poucos carvões identificáveis (Tab. 3). Regista-se, novamente, a presença de Quercus de folha caduca e perene e Leguminosae.

A metodologia de análise antracológica deste estudo envolveu também o registo de alterações anatómicas/dendrológicas como a vitrificação. A presença desta alteração no conjunto identificado foi bastante irregular, apesar de se ter registado em todas as amostras. Proporcionalmente ao total de carvões analisados, sobressaem os fragmentos de 
Quercus perenifólia da amostra 13A (com 96\% de presença desta alteração) e de Quercus caducifólio na amostra 14A com cerca de $60 \%$. Relembre-se que estas amostras foram recolhidas manualmente e que estas foram as únicas espécies nelas identificadas. Os fragmentos de Fraxinus sp. identificados na amostra 5A apresentam também cerca de 30\% desta alteração.

Em contraste com a vitrificação, a presença de fissuras radiais foi residual nos fragmentos de carvão observados. No entanto, dois casos sobressaem: Leguminosae identificadas na amostra 4A e os Fraxinus sp. da amostra 5A, com presenças de $30 \%$ e $20 \%$, respetivamente. As restantes mantiveram-se percentualmente abaixo dos $5 \%$.

Nenhuma outra característica anatómica/ dendrológica surgiu em número significativo de fragmentos analisados carvões.

Tab. 3. Resultados do conjunto antracológico.

Tab. 3. Results from charcoal analysis.

\begin{tabular}{|c|c|c|c|c|c|c|c|c|c|c|c|c|c|c|c|}
\hline Amostra & $1 \mathrm{~A}$ & $2 A$ & $3 A$ & 4A & $5 A$ & 7A & $8 \mathrm{~A}$ & $6 A$ & $9 A$ & $10 \mathrm{~A}$ & \multirow{2}{*}{\multicolumn{4}{|c|}{\begin{tabular}{|lllll|}
$11 A$ & $12 A$ & $13 A$ & $14 A$ \\
\multicolumn{2}{|c|}{} &
\end{tabular}}} & \\
\hline Fase & \multicolumn{10}{|c|}{ II } & & & & & \\
\hline Contexto & \multicolumn{7}{|c|}{$\begin{array}{c}\text { Reforço sistema de } \\
\text { contrafortagem }\end{array}$} & Muralha & \multicolumn{2}{|c|}{$\begin{array}{l}\text { Assentamento do } \\
\text { sist. contrafortagem }\end{array}$} & \multirow{2}{*}{\multicolumn{4}{|c|}{\begin{tabular}{|c|}
$\begin{array}{c}\text { Depósitos de } \\
\text { nivel. do geológico }\end{array}$ \\
$\mathbf{1 3 0}$ \\
\end{tabular}}} & \\
\hline U.E. & 108 & 109 & 110 & 111 & 114 & 125 & 127 & 121 & 128 & 129 & & & & & Total \\
\hline Alnus/Corylus sp. & & & 2 & & & 10 & 3 & & 1 & 1 & & & & & 17 \\
\hline Arbutus unedo & & & & & & 1 & & & & 6 & 2 & & & & 9 \\
\hline Castanea sativa & 20 & 5 & & & 2 & 2 & & & & & & & & & 29 \\
\hline Cistus sp. & & & & & 1 & & & & & 2 & & & & & 3 \\
\hline Erica $\mathrm{sp}$. & & & 2 & & & & & & & & & & & & 2 \\
\hline Ficus carica & & & & & & 19 & & & & & & & & & 19 \\
\hline Fraxinus sp. & & & 7 & & 54 & 3 & & & & 2 & & & & & 66 \\
\hline Juglans regia & 3 & 3 & 1 & 4 & 17 & & & & & & & & & & 28 \\
\hline Laurus sp. & & 2 & & & & 1 & & & & & & & & & 3 \\
\hline Leguminosae & 6 & 48 & 46 & 74 & 8 & 14 & 13 & 3 & 1 & 9 & 9 & & & & 231 \\
\hline Pteridium aquilinum & & & & & & & & & & & 16 & & & & 16 \\
\hline Prunus sp. & & & 2 & & & & & & & & & & & & 2 \\
\hline Quercus caducifólia & 19 & 42 & 23 & 54 & 37 & 15 & 9 & 11 & 2 & 29 & 94 & 49 & & 110 & 494 \\
\hline Quercus perenifólia & 2 & 13 & 18 & 6 & & 4 & 18 & 2 & 10 & 6 & 5 & & 132 & & 216 \\
\hline Quercus suber & & & 2 & & 7 & 3 & 2 & & & 6 & & & & & 20 \\
\hline Quercus sp. & 11 & 12 & 29 & 1 & 9 & 32 & 50 & & 30 & 23 & 6 & & 6 & & 209 \\
\hline Fagaceae & 3 & 2 & 40 & 2 & 1 & 51 & & & 11 & 49 & & & 10 & & 169 \\
\hline Rosaceae Maloidea & & 1 & 1 & & 1 & 11 & & & & & & & & & 14 \\
\hline Salix/Populus & & & & & & & & & & & 1 & & & & 1 \\
\hline Sambucus sp. & & & & 2 & 9 & 2 & 1 & & & & & & & & 14 \\
\hline Taxus baccata & & & & & & & 1 & & & & & & & & 1 \\
\hline cf. U/mus & & & & & & 1 & & & & & & & & & 1 \\
\hline Vitis vinifera & & & & & 2 & 1 & & & & & & & & & 3 \\
\hline Dicotiledónea & 10 & 22 & 27 & 7 & 57 & 28 & 53 & 5 & 45 & 17 & 22 & 1 & 1 & 1 & 296 \\
\hline Indeterminado & & & & & & 2 & & & & & & & 1 & & 3 \\
\hline TOTAL & 74 & 150 & 200 & 150 & 205 & 200 & 150 & 21 & 100 & 150 & 155 & 50 & 150 & 111 & 1866 \\
\hline
\end{tabular}

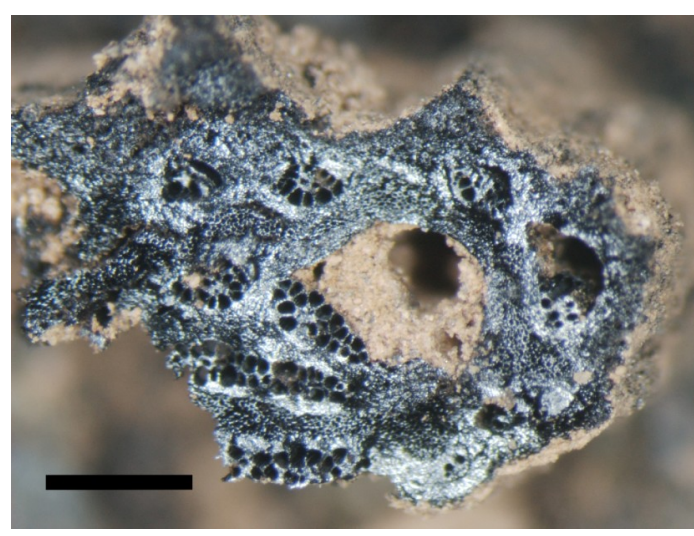

Fig. 7. Secção transversal de Pteridium aquilium (feto). Escala: $500 \mu \mathrm{m}$

Fig. 7. Transverse section of Pteridium aquilium (fern). Scale: $500 \mu \mathrm{m}$.

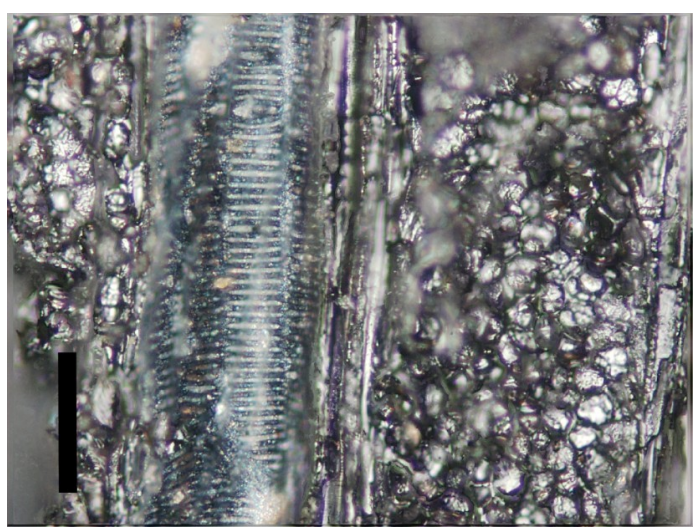

Fig. 8. Secção radial de Vitis vinifera (videira). Escala $100 \mu \mathrm{m}$.

Fig. 8. Radial section of Vitis vinifera (vine tree). Scale: $100 \mu \mathrm{m}$. 


\section{DISCUSSÃO E CONCLUSÕES}

No que respeita à Fase I identificada na sondagem 1 do castro de Cidadelhe, a interpretação conjunta dos dados carpológicos e antracológicos parecem indicar que a u.e. [130], a única unidade sedimentar desta fase que foi amostrada apresenta características sem paralelos nos restantes contextos sedimentares amostrados. Também do ponto de vista estratigráfico este contexto se distingue dos restantes pois esta unidade formou-se numa fase anterior à construção da muralha definida na Sondagem 1. Foi neste nível sedimentar que assentou a referida estrutura, pelo que o seu topo foi afetado por ação humana direta. Foi precisamente a recolha feita neste nível superior onde se verificou uma maior diversidade de frutos e sementes (Tab. 2). Apesar dos problemas de diagnóstico taxonómico acima mencionados (vide 4.1), o conjunto carpológico aparenta ser homogéneo, traduzindo um ambiente antropizado. As espécies e géneros identificados apresentam atualmente uma distribuição associada a ambientes ruderais (em taludes e muros ou bordeando caminhos) e terrenos agrícolas abandonados, surgindo também como infestantes de campos de cultivo. Alguns taxa, tais como Polygonum persicaria, Plantago, Carex e Ranunculus parecem preferir ambientes com alguma humidade edáfica.

Quanto aos dados antracológicos referentes a este contexto (vide 4.2), o reduzido leque de taxa é consentâneo com os dados provenientes do estudo carpológico, espelhando um terreno inculto, com vegetação arbustiva, fetos e esporádicas presenças de carvalhos. Haverá, no entanto, que ter em conta o facto das amostras 12A, 13A e 14A terem sido recolhidas manualmente. A existência de uma só espécie por amostra (Quercus caducifólio no caso da 12A e 14A e Quercus perenifólio na 13A) levou à conclusão de que se trata de um fenómeno de concentração devendo tratar-se de uma fragmentação de carvões provenientes dos mesmos ramos. Esta suspeita foi ainda mais consubstanciada pela similitude dos fragmentos observados, nomeadamente ao nível das alterações anatómicas observadas (presença de vitrificação, erosão e fissuras radiais), mas também no que respeita à curvatura dos anéis (exclusivamente débeis).

Desta fase mais antiga, salienta-se o conjunto de carvões, frutos e sementes recolhidos no nível superior da UE [130]. Estes parecem resultar de um evento de combustão anterior à construção da muralha, embora seja impossível determinar a distância temporal entre os dois acontecimentos. Também não é possível avaliar a extensão espacial da combustão pois os dados referem-se a uma só amostra, recolhida numa pequena área de escavação. Seja como for, os dados sugerem que antes da construção da muralha a área encontrava-se já bastante antropizada mas, muito provavelmente, inculta.
Consideramos, porém, ser necessário continuar a proceder à recolha e análise de amostras sedimentares de contextos limítrofes em futuras campanhas, de forma a confirmar ou negar esta hipótese.

Na Fase II, compreendendo os níveis interpretados como sedimentos de assentamento do sistema de contrafortagem, sedimentos de reforço do sistema de contrafortagem e troço da muralha, tanto os dados carpológicos como antracológicos (Tab. 2 e 3) apontam para a existência de plantas cultivadas. No caso da carpologia, surgem vestígios de cereais, ainda que bastante escassos, salientando-se duas bases de gluma de T. dicoccum.

Muito mais diverso que na fase anterior, o conteúdo antracológico analisado permite inferir da utilização de material lenhoso proveniente de diferentes habitats.

Destaca-se, numa primeira análise, a presença de taxa associados a carvalhais e bosques mistos (carvalho alvarinho ou cerquinho, sobreiro e teixo). Num segundo patamar de preferência encontram-se os matos arbustivos de leguminosas (giestas, codeços e/ou tojos), urzes, esteva e medronheiro. Adscritos a uma galeria ripícola surgem os freixos, amieiro/aveleira, sabugueiro, loureiro, salgueiro/ choupo e ulmeiro.

Não refletindo uma unidade ecológica distinta mas constituindo um grupo de muito importante análise paleoambiental no conjunto dos dados antracológicos estudados, surge o castanheiro, a figueira, a nogueira e a videira, todos estes circunscritos às amostras recolhidas nos sedimentos de reforço do sistema contrafortado. A madeira destas espécies não é muito comum nos contextos arqueológicos da região norte do país. O único sítio arqueológico com uma presença abundante de madeira carbonizada de castanheiro é Monte Mozinho (Penafiel), onde foi encontrada em níveis do Baixo-Império (VAZ 2012; VAZ \& TERESO 2012; VAZ et al. 2015). Aparece também noutros sítios arqueológicos, tais como Castelo de Matos, do Bronze final, e Ermidas, do século I d.C. (FIGUEIRAL 1990, 1996). Já a figueira surge no Cruito, no Crastoeiro e também em Penices e Ermidas, testemunhando assim a sua presença em níveis da Idade do Ferro e Época Romana no norte do país (FIGUEIRAL 1990, 1996). A madeira de nogueira é mais rara, surgindo, por exemplo, em Ermidas (século I d.C.) e Penices (Baixo-império) (FigueIRAL 1990, 1996).

Assim, embora o castanheiro, a figueira, a nogueira e a videira sejam autóctones (MUÑOZSOBRINO et al. 1997; BUXÓ 1997b; RAMIL REGO et al. 1998; FigueIRAL \& BETTENCOURT 2004), raramente são encontrados carvões destas espécies em sítios arqueológicos da região norte do país. Como tal, poder-se-á considerar a sua presença conjunta nas amostras como indicadora da adoção de práticas de arboricultura (BUXÓ 1997b; DESPRAT et al. 2003; TEIRA BRIÓN 2010). 
De salientar que na tabela 3 não foram considerados entre os taxa potencialmente cultivados, os carvões de rosáceas, tanto de Prunus sp. como de Rosaceae Maloideae. Embora estes tipos morfológicos incluam espécies domésticas, tais como o pessegueiro, cerejeira e ameixeira, no primeiro caso, e a macieira e pereira, no segundo, a verdade é que é grande o conjunto de espécies silvestres das quais esses carvões poderiam advir. Nota-se, contudo, que os poucos carvões destas rosáceas foram recolhidos no mesmo contexto onde todos os carvões de espécies provavelmente domésticas foram recolhidos.

$\mathrm{O}$ estudo arqueobotânico realizado permitiu não só caracterizar sumariamente duas fases cronológicas e ecológicas distintas, consentâneas com a realidade arqueológica detetada estratigraficamente, mas também forneceu bases factuais interessantes para a compreensão estratigráfica do sítio. Ambas estas realidades remetem para ambientes antropizados, pese embora com características distintas. Estas características podem traduzir no entanto um significado espacial distinto: um de âmbito muito localizado, na fase mais antiga, remetendo para o local arqueológico prévio à edificação da estrutura muralhada, e um outro, com um significado espacial difícil de determinar, relacionado com a recolha de lenha e conectado não só com espécies silvestres mas também com eventuais práticas de silvicultura. Apesar de ter sido analisado um volume limitado de sedimento, os resultados obtidos atestam o potencial deste tipo de estudos e o papel determinante que podem ter para a compreensão de aspetos cruciais da história do local e das comunidades que aí habitaram.

Urge, por ora, continuar o estudo arqueológico do sítio arqueológico, através da promoção de novas campanhas de escavação. Estas serão cruciais para a compreensão do conjunto estrutural e da sequência estratigráfica do local. Continuando a abordagem interdisciplinar iniciada, incluindo novos estudos paleoambientais, poderão acrescentar dados muito importantes para a compreensão da história do vale do Douro na Idade do Ferro e Época Romana.

\section{BIBLIOGRAFIA}

ANDERBERG, A.-L. 1994. Atlas of seeds and small fruits of Northwest-European plant species with morphological descriptions. 4. Resedaceae-Umbelliferae, Stockholm, Swedish Museum of Natural History.

BERGgren, G. 1981. Atlas of seeds and small fruits of Northwest-European plant species with morphological descriptions. 3. Salicaceae-Cruciferae, Stockholm, Swedish Museum of Natural History.

Buxó, R. 1997a. Arqueología de las Plantas. Barcelona. Crítica.

Buxó, R. 1997b. Presence of Olea europaea and Vitis vinifera in archaeological sites from the Iberian Peninsula. Lagascalia 19, 271-282.
CARRIÓN, Y. 2003. Afinidades y diferencias de las secuencias antracológicas en las vertientes mediterranea e atlantica de la peninsula Iberica. Tesis Doctoral, Facultat de Geografia i História de la Universidad de Valência.

CARRIÓN, Y. 2007. Woodland in the middle Ebro valley (Spain): dendrological analysis of archaeological timber from Bell Baker and Iron Age periods. $\mathrm{Ar}$ cheoSciences revue d'archéométrie 31: 151-161.

Desprat, S.; GoÑI, M. F. S. \& Loutre, M-F. 2003. Revealing climatic variability of the last three millennia in northwestern Iberia using pollen influx data. Earth and Planetary Science Letters, 213: 63-78.

FigueIRAL, I. 1990. Le nord-ouest du Portugal et les modifications de l'ecosysteme, du Bronze final à l'époque romaine, d'aprés l'anthracoanalyse de sites archéologiques. These Doctorat, Université des Sciences et Techniques du Languedoc.

FigUeIRAL, I. 1996. Wood resources in north-west Portugal: their availability and use from the late Bronze Age to the Roman period. Vegetation History and Archaeobotany, 5: 121-129.

Figueiral, I. \& BetTencourt, A.S., 2004. Middle/Late Bronze Age plant communities and their exploitation in the Cávado Basin (NW Portugal) as shown by charcoal analysis: the significance and cooccurrence of Quercus (deciduous) - Fabaceae. Vegetation History and Archaeobotany 13: 219232.

HENRY, A., 2011. Paléoenvironnements et gestion des combustibles au Mésolithique dans le sud de la France: anthracologie, ethnoarchéologie et expérimentation. Université Nice Sophia Antipolis.

HeIss A. 2002. Anatomy of European and North American woods - an interactive identification key, http:// www.holzanatomie.at/.

Hillman, G.; Mason, S.; De Moulins, D. \& Nesbitt, M. 1996. Identification of archaeological remains of wheat: the 1992 London workshop. Circaea 12:195 $-210$

JACOMET S. 2006. Identification of cereal remains from archaeological sites. Archaeobotany Lab, IPAS, Basel University.

LEMOS, P. 2014. As muralhas do castro de Cidadelhe (Mesão Frio, Portugal) in P. FuEnTEs, J. C. SASTRE, M. VÁZQUEZ E Ó. RodRÍGUEZ (Coord) Fortificaciones en la edad del hierro: Control de los recursos y el territorio, Zamora, La Ergástula: 299306.

LimA, A. M. C. 2010-2011. Povoamento e Organização do Território do Baixo Douro na Época da Monarquia Asturiana. Portvgalia, Nova Série, 31-32: 83-114.

LóPEZ-DóRIGA, I. 2014. Por qué datar carporrestos arqueológicos por radiocarbono? Nailos, 1:167-180.

Marguerie, D. \& Hunot, J.-Y. 2007. Charcoal analysis and dendrology: data from archaeological sites in north-western France. Journal of Archaeological Science, 34: 1417-1433.

McParland, L.C.; Collinson, M.E.; ScotT, A.C.; CAMPBELL, G. \& VEAL, R. 2010. Is vitrification in charcoal a result of high temperature burning of wood? Journal of Archaeological Science, 37: 2679-2687. 
MuÑoz Sobrino, C.; RAMIL-Rego, P. \& RodríGuez GuITIÁN, M. 1997. Upland vegetation in the north-west Iberian peninsula after the last glaciation: Forest history and deforestation dynamics. Vegetation History and Archaeobotany, 6: 215-233.

QueIroz, P. \& VAN DER Burgh, C. 1989. Wood Anatomy of Iberian Ericales. Revista de Biologia, 14: 95-134.

RAMIL REgO, P.; MUÑOZ-SOBRINO, C.; RodríGUEZGuitián, M. \& Gómez-OrellanA, L. 1998. Differences in the vegetation of the North Iberian Peninsula during the last 16,000 years. Plant Ecology, 138: 41-62.

SCHWEINGRUBer F. H. 1990a. Anatomy of European woods. Paul Haupt and Stuttgart Publishers.

SChWEINGRUBER F. H. 1990b. Microscopic Wood Anatomy. Swiss Federal Institute for Forest, Snow and Landscape Research.

Silva, A. C. F. 1986. A Cultura Castreja no Noroeste de Portugal. Câmara Municipal de Paços de Ferreira, Museu Arqueológico da Citânia de Sanfins.

TEIRA BRIÓN, A. 2010. Wild fruits, domesticated fruits. Archaeobotanical remains from the roman salwork at O Areal, Vigo (Galicia, Spain). In: C. Delhon, I. Théry-Parisot, S (Coord.), Des hommes et des plantes. Exploitation du milieu et gestion des ressources végétales de la préhistoire à nos jours. (XXXe rencontres internationales d'archéologie et d'histoire d'Antibes). Thiébault Éditions APDCA, Antibes: 199-207.

TERESO J. 2007. Paleoetnobotânica do povoado romano da Terronha de Pinhovelo (NE Transmontano). Dissertação de Mestrado, Faculdade de Ciências da Universidade do Porto.

THÉRY-PARISOT, I. \& HENRY, A. 2012. Seasoned or green? Radial cracks analysis as a method for identifying the use of green wood as fuel in archaeological charcoal. Journal of Archaeological Science 39: 381-388.

VAZ, F. C. 2012. Gestão e Usos de Recursos Vegetais no Noroeste Peninsular: a Antracologia de Monte Mozinho. Tese de Mestrado, Faculdade de Letras da Universidade do Porto.

VAZ, F. C. \& Tereso, J. P. 2012. Estudo Antracológico do compartimento 1, setor A-2008 de Monte Mozinho. Estudos do Quaternário, 8: 38-47.

Vaz, F. C.; Tereso, J. P. \& de Carvalho, T. P. 2005. Selection of firewood in Monte Mozinho (NW Iberia) in the Late Antiquity: A question of function and availability, Quaternary International (2015). http://dx.doi.org/10.1016/j.quaint.2015.10.041

Vernet J.-L.; Ogereau, P.; Figueiral, I.; Machado YANEs, C. \& UzQuiano, P. 2001. Guide d'identification des charbons de bois préhistoriques et récents. Sud-ouest de l'Europe :France,Péninsule ibérique et Îles Canaries. Paris, CNRS Editions. 